\title{
Barrett Adenocarcinoma
}

National Cancer Institute

\section{Source}

National Cancer Institute. Barrett Adenocarcinoma. NCI Thesaurus. Code C7027.

An adenocarcinoma arising from Barrett metaplastic epithelium in the esophagus. There is evidence supporting the idea that the Barrett adenocarcinomas develop through a stepwise progression through intestinal metaplastic epithelium to epithelial dysplasia to malignancy. Adenocarcinomas arising in the setting of Barrett esophagus are typically papillary and/or tubular. In terms of grading, they are well or moderately differentiated adenocarcinomas. -- 2002 